\title{
Non-extensive radiobiology
}

\author{
O. Sotolongo-Grau*, D. Rodriguez-Perez*, J. C. Antoranz ${ }^{*, \dagger}$ and O. \\ Sotolongo-Costa ${ }^{\dagger}$ \\ *UNED, Departamento de Física Matemática y de Fluidos \\ ${ }^{\dagger}$ UH, Cátedra de Sistemas Complejos Henri Poincaré
}

\begin{abstract}
The expression of survival factors for radiation damaged cells is based on probabilistic assumptions and experimentally fitted for each tumor, radiation and conditions. Here we show how the simplest of these radiobiological models can be derived from the maximum entropy principle of the classical Boltzmann-Gibbs expression. We extend this derivation using the Tsallis entropy and a cutoff hypothesis, motivated by clinical observations. A generalization of the exponential, the logarithm and the product to a non-extensive framework, provides a simple formula for the survival fraction corresponding to the application of several radiation doses on a living tissue. The obtained expression shows a remarkable agreement with the experimental data found in the literature, also providing a new interpretation of some of the parameters introduced anew. It is also shown how the presented formalism may has direct application in radiotherapy treatment optimization through the definition of the potential effect difference, simply calculated between the tumour and the surrounding tissue.
\end{abstract}

Keywords: Radiobiology, Survival fraction, Entropy

PACS: 05.20.-y, 87.10.-e, 87.53.Ay, 87.55.dh

\section{INTRODUCTION}

One of the main concerns of a radiation oncologist is to find a treatment which, maximizing the damage over the tumor, minimizes it over the surrounding healthy tissue. In order to reach a suitable treatment the radiobiologists have developed some empirical models describing the interaction between radiation and living tissues (see [1] for a review of radiobiology models) capable of finding the survival fraction, $F_{s}$, of cells under a radiation dose, $D$. These models applicability limits are not clear so multiple corrections have been developed in order to fit the experimental data [2].

The concept of tissue effect, $E$, raised from some of these models [3] is used to compare different treatments each other. Usually expressed as $E=-\ln \left(F_{S}\right)$ is a dimensionless magnitude that gathers several models of interaction between cells and ionizing radiation.

The simplest radiobiology model is the linear one. Here the tissue effect is considered linear to the radiation dose, $E=\alpha D$, and the survival fraction, $F_{s}=\exp (-\alpha D)$, is viewed as the cumulative survival probability of a cell under any dose below $D$. This probability fullfils the additive property meaning that the effects of radiation are cumulative following an additive model and the survival fraction for two doses could be found as $F_{S}\left[D_{1}+D_{2}\right]=F_{S}\left[D_{1}\right] \cdot F_{S}\left[D_{2}\right]$.

However, this model only fits the experimental data for some tissues, under low radiation doses [1], so the tissue effect must be corrected to $E=\alpha D+\beta D^{2}$, called the 
linear quadratic (LQ) model. But then the survival fraction loses the additive property, $F_{S}\left[D_{1}+D_{2}\right]<F_{S}\left[D_{1}\right] \cdot F_{S}\left[D_{2}\right]$, and the tissue effect becomes a supperadditive quantity, $E\left[D_{1}+D_{2}\right]>E\left[D_{1}\right]+E\left[D_{2}\right]$.

As a result of the nonlinear nature of $E$ in this case, the superposition principle is not fulfilled. However any model of interaction between radiation and living tissues must allow to divide a continuous radiation in finite intervals and the resultant tissue effect must be the same.

Indeed, it is easy to show that, under the LQ viewpoint, if the tissue effect were additive for different radiation sessions, then the additivity of the dose would not hold. Conversely, assuming that the dose is additive then the tissue effect is not equivalent to the sum of the effects for different doses. This result suggests that the radiobiological problem must be approached from a non extensive formulation [4].

In this work we use at the first stage the Boltzmann-Gibbs (BG) entropy in order to find the expression of the tissue effect as a function of the absorbed dose. Later, along with the Tsallis entropy [5] definition, it is assumed that a critical value of the radiation dose kills every single cell and a general expression for survival fraction is found. This survival fraction expression fits the experimental data even where previous empirical models fail. Using the $q$-deformed functions $[6,7]$ a new expression to find the survival fraction of a whole treatment is found allowing to show hints to find the best treatment.

\section{FIRST STEP: THE CLASSICAL APPROACH}

First we study the extensive problem applying the BG entropy (in units of the Boltzmann constant),

$$
S=-\int_{0}^{\infty} \ln [p(E)] p(E) d E,
$$

where in this case $E$ is, as before, the tissue effect and $p(E)$ is the cell killing probability density.

According to the maximum entropy principle, if $p(E)$ satisfies the normalization condition and a finite mean value of the tissue effect does exist, then the problem of finding the $p(E)$ that extremizes the BG entropy under the above conditions can be posed. It is well known that among all continuous probability distributions for a positive continuous variable with a fixed mean value, the exponential distribution has the largest entropy [8]. So,

$$
p(E)=\frac{1}{\langle E\rangle} e^{-\frac{E}{\langle E\rangle},}
$$

and the survival probability of a single cell will be

$$
F_{s}=\int_{E}^{\infty} p(x) d x=e^{-\frac{E}{\langle E\rangle}} .
$$

The survival probability here must fulfill the dose additivity property. This can be achieved if, following the discussion in the previous section, the tissue effect is proportional to the absorbed dose:

$$
E=\alpha_{0} D,
$$


where $\alpha_{0}$ is chosen as a constant that makes $E$ adimensional.

It must be noted that (3) is the experimentally proved and currently used expression for the survival fraction as a function of tissue effect and justified in the literature only through empirical arguments [1]. We can take $\alpha=\alpha_{0} /\langle E\rangle=1 /\langle D\rangle$ and the expression (3) becomes expressed in the known standard radiobiology form of the linear model.

Even when the BG treatment of the problem does not cover the available data, it shows that the tissue effect must be defined as proportional to the absorbed dose of radiation. However the empiric expressions already known show, as has been discussed in the introduction, that the survival probability of a cell does not fulfill the additive property. Since this is usually associated to non extensive problems the solution must be searched using a non extensive definition of entropy. On the other hand, the Tsallis formulation of the entropy has been proved its helpfulness when applied to problems of this nature.

\section{ONE STEP FURTHER: THE GENERALIZED APPROACH}

To apply the maximum entropy principle, in the Tsallis version, to the problem of finding the survival fraction of a living tissue [9] that receives a radiation, we postulate the existence of some amount of absorbed radiation $D_{0}<\infty$ (or its equivalent "minimal annihilation effect", $E_{0}=\alpha_{0} D_{0}$ ) after which no cell survives. The application of the maximum entropy principle performs like the usual one but with a few modifications.

The Tsallis entropy becomes

$$
S_{q}=\frac{1}{q-1}\left(1-\int_{0}^{E_{0}} p^{q}(E) d E\right)
$$

the normalization condition is in this case $\int_{0}^{E_{0}} p(E) d E=1$ and the $q$-mean value becomes $\int_{0}^{E_{0}} p^{q}(E) E d E=\langle E\rangle_{q}<\infty$. With this definition, all properties of the tissue and its characteristics of the interaction with radiation become included in $\langle E\rangle_{q}$ and therefore in $E_{0}$. This is the only parameter (besides $q$ ) entering in our description. It is clear that the determination of $\langle E\rangle_{q}$ for the different tissues under different conditions of radiation would give the necessary information for the characterization of the survival factor.

To calculate the maximum of (5) under the above conditions the well known method of Lagrange multipliers [7] is applied, obtaining

$$
E_{0}=\frac{2-q}{1-q}\left(\frac{\langle E\rangle_{q}}{2-q}\right)^{\frac{1}{2-q}}
$$

and

$$
p(E)=\left(\frac{2-q}{\langle E\rangle_{q}}\right)^{\frac{1}{2-q}}\left(1-\frac{1-q}{2-q}\left(\frac{2-q}{\langle E\rangle_{q}}\right)^{\frac{1}{2-q}} E\right)^{\frac{1}{1-q}}
$$


Then the survival factor is

$$
F_{S}(E)=\int_{E}^{E_{0}} p(x) d x=\left(1-\frac{E}{E_{0}}\right)^{\frac{2-q}{1-q}}
$$

with $q<1$ for $E<E_{0}$ and zero otherwise. It is not hard to see that when $q \rightarrow 1$ then $E_{0} \rightarrow \infty$ and $\langle E\rangle_{q} \rightarrow\langle E\rangle$.

Equation (8) can be written

$$
F_{S}(D)=\left\{\begin{array}{ll}
\left(1-\frac{D}{D_{0}}\right)^{\gamma} & \forall D<D_{0} \\
0 & \forall D \geqslant D_{0}
\end{array},\right.
$$

where we introduced $E=\alpha_{0} D, \gamma=\frac{2-q}{1-q}$ and $D_{0}=E_{0} / \alpha_{0}$. Finally, the LQ model is easily recovered from (9) in the limit $q \rightarrow 1$ up to order two in a Taylor series expansion [10].

\section{Tsallis based Survival fraction properties}

The linear model for the tissue effect [1] implies that if the dose is additive the corresponding survival fraction is multiplicative. Though this property belongs only to the linear model and not to more general descriptions like the LQ model [1] and others, we think it is worth to find a link between the additivity property of the dose and the probabilistic properties of the cell survival fraction.

Let us define the $\exp _{\gamma}(x)$ function

$$
\exp _{\gamma}(x)=\left[1+\frac{x}{\gamma}\right]^{\gamma}
$$

and the $\ln _{\gamma}(x)$ its inverse function

$$
\ln _{\gamma}\left(\exp _{\gamma}(x)\right)=x
$$

Then, let us introduce the $\gamma$-product of two numbers $x$ and $y$ as

$$
x \otimes_{\gamma} y=\exp _{\gamma}\left[\ln _{\gamma}(x)+\ln _{\gamma}(y)\right]=\left[x^{\frac{1}{\gamma}}+y^{\frac{1}{\gamma}}-1\right]^{\gamma} .
$$

Note that definitions (10) and (11) are not essentially different from the $q$-exponential and $q$-logarithm presented in [6]. We are just introducing these definitions to simplify the calculations.

Let us now define the "generalized tissue effect" as $E=-\frac{E_{0}}{\gamma} \ln _{\gamma}\left(F_{s}\right)$. We demand this effect to satisfy the additive property. Then the survival fraction expressed as $F_{S}=$ $\exp _{\gamma}\left(-\gamma \frac{E}{E_{0}}\right)=\exp _{\gamma}\left(-\gamma \frac{D}{D_{0}}\right)$, becomes $\gamma$-multiplicative. This implies that the statistical independence of the survival fractions is only possible when $\gamma \rightarrow \infty(q \rightarrow 1)$.

The survival fraction for the sum of the effects after $N$ doses becomes 


$$
F_{S}(N E)=\left[1-\sum_{i=1}^{N} \frac{E_{i}}{E_{0}}\right]^{\gamma}=\exp _{\gamma}\left[-\sum_{i=1}^{N} \gamma \frac{E_{i}}{E_{0}}\right]=\left[\bigotimes_{i=1}^{N}\right]_{\gamma} F_{S}\left(E_{i}\right)
$$

where $\left[\otimes_{i=1}^{N}\right]_{\gamma}$ denotes the iterated application of the $\gamma$-product.

\section{EXPERIMENTAL AGREEMENT}

Equation (9) represents the survival fraction in terms of the measurable quantities $D$ (radiation dose) and $D_{0}$ (minimal annihilation dose). In order to compare our model with the experimental data we have selected some survival curves from the literature where the survival fraction $F_{S}$ is represented as a function of $D$ for different radiation conditions. However, if $D$ is rescaled as $1-D / D_{0}$, as usual in phase transition phenomena, all curves corresponding to the same tissue collapse to the same straight line in a log-log plot.

The expression of $\ln \left[F_{S}\right]$ has been fitted for 23 experimental data sets, corresponding to 5 different tissues, in terms of the rescaled variable $\ln \left[1-D / D_{0}\right]$, minimizing the appropriate least squares functional using the steepest descent method [11]. The slope of these lines are the values of $\gamma$ meaning that $D_{0}$ is the natural unit of $D$.

Figure 1 shows, in a log-log plot, the comparison of our model with all these data sets. In order to represent all data sets in the same plot the survival fraction is shown normalized by $\gamma$ as $\left(F_{S}\right)^{1 / \gamma}$.

All the information about the kind of radiation, radiation rate, etc. is contained in the phenomenological term $D_{0}$, whereas tissues are characterized by $\gamma$. This makes (9) a very general expression with universal characteristics since the phase transition described by (9) is homomorphic with the phase transition of ferromagnets near the Curie point [17]. The exponent $\gamma$ in this case, as in ferromagnetic phase transitions, determines the universality class. Then $\gamma$ in our case deals only with the kind of tissue that interacts with radiation [10].

\section{IS IT POSSIBLE TO DESIGN THE BEST PROTOCOL?}

The expression for the survival fraction after $N$ doses of radiation must allow to compare the damage provoked on the tumor and surrounding tissue cells if the magnitudes $\gamma$ and $D_{0}$ are known for both, tumor and tissue. Let us assume we know the recommended radiation dose $D_{+}$per session for a treatment with $N$ sessions at several values of $N$. We will define the potential effect as

$$
\chi=-\ln \left[F_{S}\right],
$$

and then the recommended potential effect per dose over the tumor will be,

$$
\chi_{+}=-\gamma^{(t)} \ln \left[1-\frac{D_{+}}{D_{0}^{(t)}}\right]
$$




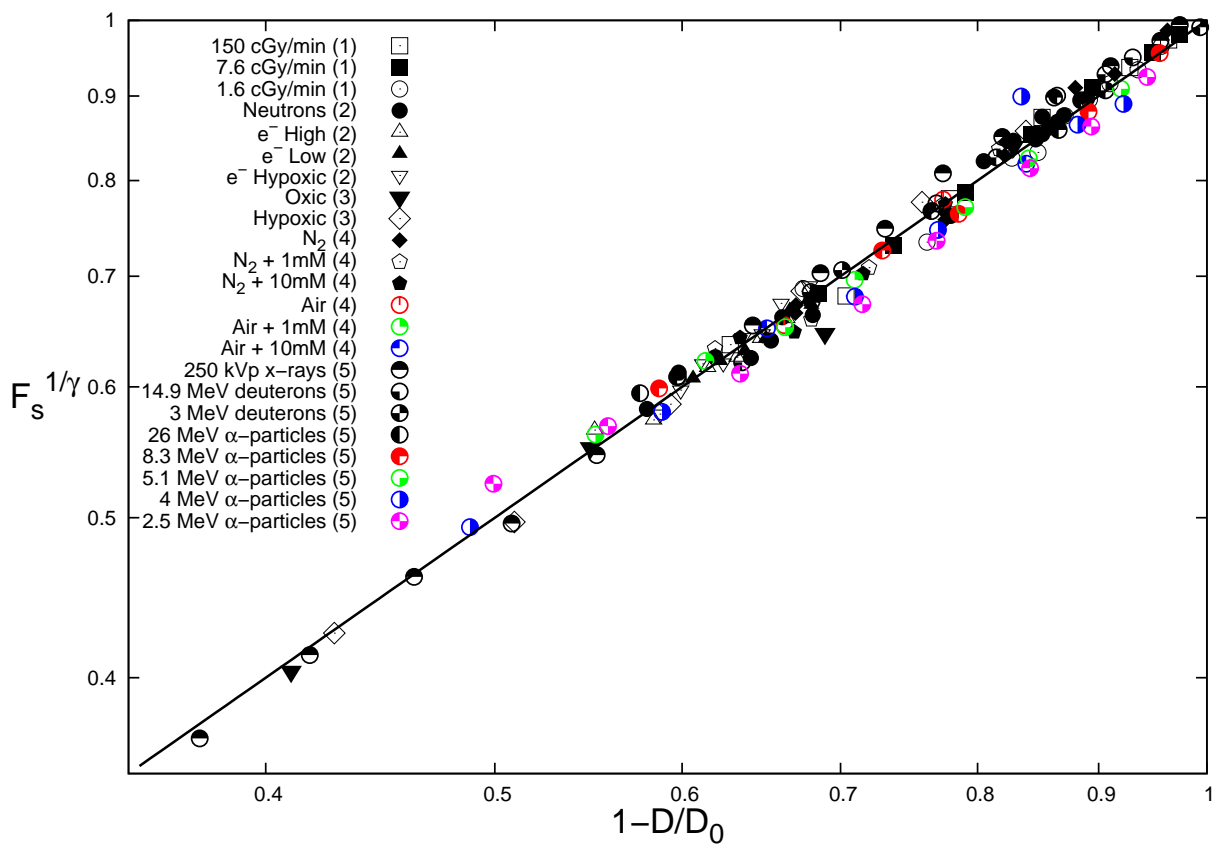

FIGURE 1. Normalized survival fractions, $\left(F_{s}\right)^{1 / \gamma}$, as a function of the rescaled radiation dose, $1-$ $D / D_{0}$. The straight line shown is $y=x$. Values for $\gamma$ and $D_{0}$ are detailed in table 1 .

where $\gamma^{(t)}$ and $D_{0}^{(t)}$ are the characteristic radiation coefficients for the tumor. If we have already characterized the healthy tissue around the tumor with $\gamma^{(h)}$ and $D_{0}^{(h)}$ then we can find the survival fraction of cells after the treatment for the tumor,

$$
\Theta^{(t)}=\exp _{\gamma^{(t)}}\left[N \ln _{\gamma^{(t)}}\left[\exp \left(-\chi_{+}\right)\right]\right],
$$

and the healthy tissue,

$$
\Theta^{(h)}=\exp _{\gamma^{(h)}}\left[\frac{\gamma^{(h)} D_{0}^{(t)}}{\gamma^{(t)} D_{0}^{(h)}} N \ln _{\gamma^{(t)}}\left[\exp \left(-\chi_{+}\right)\right]\right]
$$

In order to find the best treatment all we need is to calculate the difference of potentials for the treatment,

$$
\Delta \chi=\chi^{(t)}-\chi^{(h)}=\ln \left[\frac{\Theta^{(h)}}{\Theta^{(t)}}\right],
$$

and guarantee that it will be positive. This could be achieved taking advantage of the different responses to radiation of tumor and normal tissues. Also, if this response is too close for an specific kind of radiation or a given dose rate, those conditions could be changed looking for a higher potential difference. 


\section{CONCLUSIONS}

A new theoretical expression for the survival fraction of cells under radiation has been found, using the Tsallis formulation of entropy. The existence of a critical value for the absorbed radiation dose under which no cells survive is introduced in the formulation in order to get a proper expression. The new expression depends of two coefficients that characterize the tissue behaviour under radiation $(\gamma)$ and the specifics conditions in which the radiation is applied $\left(D_{0}\right)$.

The Tsallis mathematical formalism allows to redefine the multiplication operation giving a way to find the survival fraction after several radiation sessions. If the characteristic coefficients are known for the tumor and the surrounding tissue then some hints can be given to choose the less harmful, albeit most efficient, treatment to apply.

\section{ACKNOWLEDGMENTS}

The authors wish to thank Prof. Juan Antonio Santos, MD, for fruitful discussions. They also acknowledge the Spanish Ministerio de Industria for its support through the Proyecto CD-TEAM, CENIT.

\section{REFERENCES}

1. M. Tubiana, Introduction to Radiobiology, Taylor \& Francis, London, 1990.

2. M. C. Joiner, "The linear-quadratic approach to fractionation," in Basic Clinical Radiobiology for Radiation Oncologists, edited by G. Steel, Edward Arnold Publishers, London, 1993, pp. 55-64.

3. A. van der Kogel, and C. Arnout, "Calculation of isoeffect relationships," in Basic Clinical Radiobiology for Radiation Oncologists, edited by G. Steel, Edward Arnold Publishers, London, 1993, pp. 72-80.

4. C. Tsallis, Brazilian Journal of Physics 29, 1-35 (1999), URL arXiv:cond-mat/ 9903356v1 [cond-mat.stat-mech].

5. E. M. F. Curado, and C. Tsallis, Journal of Physics A: Mathematical and General 24, L69-L72 (1991).

6. C. Tsallis, Introduction to nonextensive statistical mechanics, Springer, New York, 2009.

7. A. Platino, and A. Plastino, Brazilian Journal of Physics 29, 50-60 (1999).

8. T. Cover, and A. Joy, Elements of Information Theory, John Wiley \& Sons, Inc., Hoboken, New Jersey, 2006.

9. G. Steel, "Clonogenic cells and the concept of cell survival," in Basic Clinical Radiobiology for Radiation Oncologists, edited by G. Steel, Edward Arnold Publishers, London, 1993, pp. 28-39.

10. O. Sotolongo-Grau, D. Rodriguez-Perez, J. Santos-Miranda, J. Antoranz, and O. Sotolongo-Costa (2009), URL arXiv:0907.5551v2 [physics .med-ph] .

11. W. Press, S. Teukolsky, W. Vetterling, and B. Flannery, Numerical Recipes in C, The Art of Scientific Computing, Cambrige, Cambrige University Press, 1992.

12. G. Steel, J. Down, J. Peacock, and T. Stephens, Radiother Oncol 5, 321-331 (1986).

13. T. Alper, Br Med Bull 29, 3-6 (1973).

14. R. Hill, R. Bush, and P. Yeung, British Journal of Radiology 44, 299-304 (1971).

15. G. Adams, "Hypoxic cell sensitizers for radiotherapy," in Cancer: A comprehensive treatise, edited by F. Baker, Plenum Press, New York, 1997, vol. 6.

16. G. Barendsen, Curr Top Radiat Res Quarterly 4, 293-356 (1968).

17. L. Landau, and E. Lifshitz, Statistical physics, Pergamon Press, 1980. 
TABLE 1. Values for $\gamma$ and $D_{0}$ obtained from the fitting with experimental data.

(1) Human melanoma $(\gamma=14.0 \pm 0.9)$ irradiated at different dose rates. Data extracted from [12].

\begin{tabular}{ll}
\hline $150 \mathrm{cGy} / \mathrm{min}$ & $D_{0}=27 \pm 2 \mathrm{~Gy}$ \\
\hline $7.6 \mathrm{cGy} / \mathrm{min}$ & $D_{0}=38 \pm 4 \mathrm{~Gy}$ \\
\hline $1.6 \mathrm{cGy} / \mathrm{min}$ & $D_{0}=46 \pm 5 \mathrm{~Gy}$ \\
\hline
\end{tabular}

(2) Intestinal stem-cells $(\gamma=30.5 \pm 0.4)$ irradiated with different particles an conditions. Data extracted from [13].

\begin{tabular}{lr}
\hline Neutrons & $D_{0}=36.0 \pm 0.7 \mathrm{~Gy}$ \\
\hline Electrons (high dose rate) & $D_{0}=62.2 \pm 1.2 \mathrm{~Gy}$ \\
\hline Electrons (low dose rate) & $D_{0}=68.8 \pm 1.6 \mathrm{~Gy}$ \\
\hline Electrons (hypoxic conditions) & $D_{0}=162 \pm 3 \mathrm{~Gy}$ \\
\hline
\end{tabular}

(3) Cultured mammalian cells $(\gamma=8.9 \pm 0.6)$ exposed to $x$-rays under oxic and hypoxic conditions. Data extracted from [14].

\begin{tabular}{lr}
\hline Oxic & $D_{0}=21.7 \pm 1.4 \mathrm{~Gy}$ \\
\hline Hypoxic & $D_{0}=61 \pm 4 \mathrm{~Gy}$ \\
\hline
\end{tabular}

(4) Chinese hamster cells $(\gamma=14.2 \pm 0.6)$ irradiated in the presence or absence of misonidazole. Data extracted from [15].

\begin{tabular}{ll}
\hline Hypoxic & $D_{0}=85 \pm 7 \mathrm{~Gy}$ \\
\hline Hypoxic, $1 \mathrm{mM}$ & $D_{0}=46 \pm 3 \mathrm{~Gy}$ \\
\hline Hypoxic, $10 \mathrm{mM}$ & $D_{0}=33 \pm 2 \mathrm{~Gy}$ \\
\hline Aerated & $D_{0}=29 \pm 3 \mathrm{~Gy}$ \\
\hline Aerated, $1 \mathrm{mM}$ & $D_{0}=28 \pm 3 \mathrm{~Gy}$ \\
\hline Aerated, $10 \mathrm{mM}$ & $D_{0}=32 \pm 4 \mathrm{~Gy}$ \\
\hline
\end{tabular}

(5) Human kidney cells $(\gamma=8.8 \pm 0.3)$ exposed in vitro to radiations of different energies. Data extracted from [16].

\begin{tabular}{lr}
\hline $250 \mathrm{kVp}$ x-rays & $D_{0}=22.4 \pm 0.8 \mathrm{~Gy}$ \\
\hline $14.9 \mathrm{MeV}$ deuterons & $D_{0}=22 \pm 3 \mathrm{~Gy}$ \\
\hline $3 \mathrm{MeV}$ deuterons & $D_{0}=17 \pm 2 \mathrm{~Gy}$ \\
\hline $26 \mathrm{MeV} \alpha$-particles & $D_{0}=15 \pm 2 \mathrm{~Gy}$ \\
\hline $8.3 \mathrm{MeV} \alpha$-particles & $D_{0}=9.1 \pm 1.2 \mathrm{~Gy}$ \\
\hline $5.1 \mathrm{MeV} \alpha$-particles & $D_{0}=7.5 \pm 0.8 \mathrm{~Gy}$ \\
\hline $4 \mathrm{MeV} \alpha$-particles & $D_{0}=6.9 \pm 0.6 \mathrm{~Gy}$ \\
\hline $2.5 \mathrm{MeV} \alpha$-particles & $D_{0}=9.2 \pm 0.7 \mathrm{~Gy}$ \\
\hline
\end{tabular}

